\title{
Electric-field-induced and temperature-induced phase transitions in high-strain ferroelectric $\mathrm{Pb}(\mathrm{Mg} 1 / 3 \mathrm{Nb} 2 / 3) 0.67 \mathrm{Ti} 0.33 \mathrm{O} 3$ single crystal
}

\section{Authors: R. R. Chien, C.-S. Tu, V. Hugo Schmidt, and F.-T. Wang}

This is an author-created, un-copyedited version of an article published in Journal of Physics: Condensed Matter. IOP Publishing Ltd is not responsible for any errors or omissions in this version of the manuscript or any version derived from it. The Version of Record is available online at https://dx.doi.org/10.1088/0953-8984/18/35/018.

R.R. Chien, C.-S. Tu, V.H. Schmidt, and F.-T. Wang, "Electric-field-induced and temperature-induced phase transitions in high-strain ferroelectric $\mathrm{Pb}(\mathrm{Mg} 1 / 3 \mathrm{Nb} 2 / 3) 0.67 \mathrm{Ti} 0.33 \mathrm{O} 3$ single crystal," Journal of Physics: Condensed Matter 18, 8337-8344 (2006). doi: 10.1088/0953-8984/18/35/018.

Made available through Montana State University's ScholarWorks scholarworks.montana.edu 


\title{
Electric-field-induced and temperature-induced phase transitions in high-strain ferroelectric $\mathrm{Pb}\left(\mathrm{Mg}_{1 / 3} \mathrm{Nb}_{2 / 3}\right)_{0.67} \mathrm{Ti}_{0.33} \mathrm{O}_{3}$ single crystal
}

\author{
R R Chien ${ }^{1}$, Chi-Shun Tu ${ }^{2}$, V Hugo Schmidt ${ }^{1}$ and F-T Wang ${ }^{2}$ \\ ${ }^{1}$ Department of Physics, Montana State University, Bozeman, MT 59717, USA \\ ${ }^{2}$ Graduate Institute of Applied Science and Engineering, Fu Jen University, Taipei 242, Taiwan
}

\begin{abstract}
This work is to study electric $(E)$-field-induced and temperature-induced phase transitions in (001)-cut $\mathrm{Pb}\left(\mathrm{Mg}_{1 / 3} \mathrm{Nb}_{2 / 3}\right)_{0.67} \mathrm{Ti}_{0.33} \mathrm{O}_{3}$ (PMNT33\%) single crystal, which are critical concerns for piezoelectric applications. Dielectric properties and domain structures (by polarizing microscope) are measured as functions of temperature and $E$ field. The hysteresis loop of the polarization versus $E$ field at room temperature is also measured. Without any $E$-field application, upon heating a first-order-type phase transition sequence rhombohedral

$(\mathrm{R}) \rightarrow$ rhombohedral/monoclinic/[001]tetragonal $\left(\mathrm{R} / \mathrm{M} / \mathrm{T}_{001}\right) \rightarrow$ cubic $(\mathrm{C})$ takes place near 350 and $430 \mathrm{~K}$, respectively. Under a dc $E$-field application along [001] at room temperature, [001] tetragonal $\left(\mathrm{T}_{001}\right)$ phase domains are induced by various phase transition sequences, i.e. $\mathrm{R} \rightarrow \mathrm{T}_{001}, \mathrm{R} \rightarrow \mathrm{M} \rightarrow$ $\mathrm{T}_{001}, \mathrm{R} \rightarrow \mathrm{T} \rightarrow \mathrm{T}_{001}$, and $\mathrm{R} \rightarrow \mathrm{M} \rightarrow \mathrm{T} \rightarrow \mathrm{T}_{001}$, as the $E$-field strength increases. In addition, $E$-field-induced microcracking is observed in this work.

(Some figures in this article are in colour only in the electronic version)
\end{abstract}

\section{Introduction}

Relaxor-based ferroelectric single crystals of $\mathrm{Pb}\left(\mathrm{Mg}_{1 / 3} \mathrm{Nb}_{2 / 3}\right)_{1-x} \mathrm{Ti}_{x} \mathrm{O}_{3}$ (PMNTx) and $\mathrm{Pb}\left(\mathrm{Zn}_{1 / 3} \mathrm{Nb}_{2 / 3}\right)_{1-x} \mathrm{Ti}_{x} \mathrm{O}_{3}$ (PZNTx), which have a morphotropic phase boundary (MPB) between the $\mathrm{R}$ and $\mathrm{T}$ phases, have attracted much attention due to their high performance in piezoelectric-related applications [1-6]. These crystals exhibit piezoelectric properties about ten times better than the ceramics that are generally used, such as an extremely large electromechanical coupling factor $k_{33}(>94 \%)$, ultrahigh piezoelectric coefficients ( $>2500 \mathrm{pC} \mathrm{N}^{-1}$ ), a large strain level (up to $\sim 1.7 \%$ ), and low hysteresis [7-9]. An $E$ field poling is usually performed on these materials to enhance the piezoelectric performance before application. The ultrahigh piezoelectric response is attributed to polarization rotation 


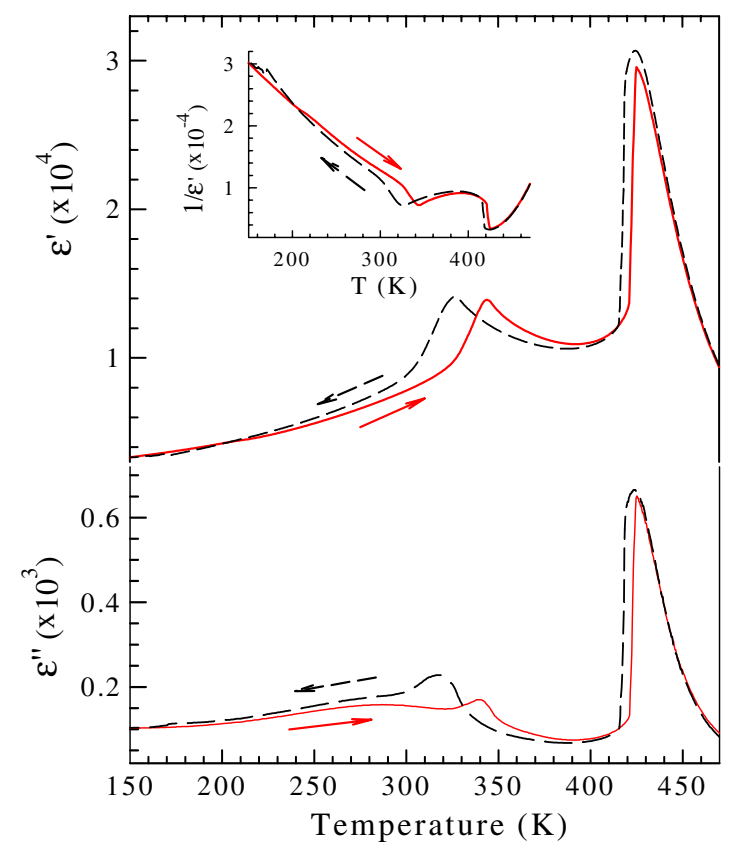

Figure 1. Temperature-dependent dielectric permittivity measured at $10 \mathrm{kHz}$ upon heating and cooling in the unpoled (001)-cut PMNT33\% crystal. The inset is the reciprocal of $\varepsilon^{\prime}$.

induced by an external dc $E$ field between the T and R phases through intermediate (M) or orthorhombic $(\mathrm{O})$ symmetries [10]. It has generated great interest in investigating phase (structural) transitions near the MPB under the application of thermal, $E$-field, and stress to understand the underlying mechanism of the piezoelectric properties [11].

In this report, we study $E$-field-induced and temperature-induced phase transitions in a (001)-cut PMNT33\% single crystal by dielectric permittivity and domain observations with polarizing microscopy. The hysteresis loop of polarization versus $E$ field was also measured at room temperature (RT). By using relations of crystallographic symmetry and optical extinction, polarizing light microscopy provides a real-time direct observation of the polarization orientations in ferroelectric domains and their corresponding crystal phases.

Physical properties of PMNTx under E-field application, such as the phase (structural symmetry) nature, depends strongly on titanium content, crystallographic orientation, strength of the external poling $E$ field, and temperature [11-14]. In order to understand the physical origin of the $E$-field-induced phase transitions, in section 3 we shall compare this result with our previous results from PMNTx crystals with various crystallographic orientation and titanium content, i.e. (001)-cut PMNT24\%, (111)-cut PMNT33\%, and (001)-cut PMNT40\% crystals [15-17].

\section{Experimental procedure}

The lead magnesium niobate-lead titanate crystal PMNT33\% was grown using a modified Bridgman method and was cut perpendicular to a $\langle 001\rangle$ direction after aligning it by $\mathrm{x}$-ray diffraction. The Ti concentration $(x \%)$ was determined from $x=\left[T_{\mathrm{m}}\left({ }^{\circ} \mathrm{C}\right)+10\right] / 5$, where $T_{\mathrm{m}}$ is the dielectric maximum temperature obtained upon heating without $E$-field poling, as shown in figure 1 [13]. Before any measurement described below, the sample was annealed 
above $200^{\circ} \mathrm{C}$. For dielectric measurements, a Wayne-Kerr Precision Analyzer PMA3260A with four-lead connections was used to obtain the real $\left(\varepsilon^{\prime}\right)$ and imaginary $\left(\varepsilon^{\prime \prime}\right)$ parts of dielectric permittivity. A Janis CCS-450 cold-head was used with a Lakeshore 340 temperature controller. The sample dimensions are $7.8 \times 3.1 \times 0.6 \mathrm{~mm}^{3}$, and gold electrodes were deposited on sample (001) surfaces by dc sputtering. Two processes were used in the dielectric measurements, called 'zero-field-heated' (ZFH) and 'zero-field-cooled' (ZFC), in which the data were taken upon heating and cooling, respectively, without any $E$-field poling. The hysteresis loop of the polarization versus $E$ field was taken at RT by using a Sawyer-Tower circuit at $f=46 \mathrm{~Hz}$.

The domain observations as functions of temperature and $E$ field were made by using a Linkam THMS600 heating/cooling stage mounted on a Nikon E600POL polarizing microscope with a $0^{\circ} / 90^{\circ}$ crossed polarizer/analyser (P/A) pair. The detailed experimental configuration can be found in [18]. The sample thickness is about $65 \mu \mathrm{m}$. Transparent conductive films of indium tin oxide (ITO) were deposited on sample (001) surfaces by dc sputtering. The $\langle 110\rangle$ orientation of the sample edge was determined by $\mathrm{x}$-ray diffraction and aligned with one of the crossed $\mathrm{P} / \mathrm{A}: 0^{\circ}$ axes so that the extinction angles shown in all domain micrographs are measured from $\langle 110\rangle$. In $E$-field-dependent domain observation, a dc $E$ field was applied along [001] at RT.

\section{Results and discussion}

\subsection{Temperature-dependent dielectric permittivity}

Figure 1 shows the temperature-dependent real part $\varepsilon^{\prime}$ and imaginary part $\varepsilon^{\prime \prime}$ of the dielectric permittivity at measuring frequency $f=10 \mathrm{kHz}$ from ZFH and ZFC, respectively. The maximum temperatures $\left(T_{\mathrm{m}}\right)$ of $\varepsilon^{\prime}(\mathrm{ZFH})$ and $\varepsilon^{\prime}(\mathrm{ZFC})$ occur at 425 and $420 \mathrm{~K}$, respectively. Obvious thermal hystereses are observed in the temperature regions $\sim 250-400 \mathrm{~K}$ and $\sim 415-430 \mathrm{~K}$, respectively. As shown in the inset of figure 1, two steep dips in the reciprocal of $\varepsilon^{\prime}$ are seen at $340(320 \mathrm{~K})$ and $425(420 \mathrm{~K})$ for ZFH (ZFC). Both steep phenomena and thermal hystereses imply two first-order-type transitions near $340 \mathrm{~K}(320 \mathrm{~K})$ and $425 \mathrm{~K}(420 \mathrm{~K})$, respectively, upon heating (cooling).

\subsection{Principle of polarizing microscopy}

Through measuring optical extinction angles from polarizing microscopy, various crystallographic symmetries (or phases) can be determined for most cases. For interpreting domain structures obtained from polarizing microscopy, a review of principles of optical extinction for the (001)-cut crystal can be found in [19]. Figure 2 shows the (001)-cut projection with all four sides folded out. The inner square outlines the front face of the cube.

In this study, the extinction angles are measured with regard to $\langle 110\rangle$, as shown in figure 2 , in which the solid crosses within the symbols represent the orientation of the extinction. Thus, $\mathrm{R}$ phase domains represented by triangles with solid crosses in figure 2 have extinction at $0^{\circ}$ (or $90^{\circ}$ ). T phase domains represented by squares with solid crosses have extinction at $45^{\circ}$. T phase domains with polarization $\boldsymbol{P}$ along the [001] axis represented by the solid black square in the centre have extinction at every orientation of the crossed P/A pair and will be written as ' $\mathrm{T}_{001}$ ' in the following discussion. The $\mathrm{O}$ phase domains represented by circles with solid crosses have extinctions at $0^{\circ}$ (or $90^{\circ}$ ) or $45^{\circ}$, whereas $\mathrm{R}$ domains only give extinctions at $0^{\circ}$. Thus, the (001)-cut shown in figure 2 clearly distinguishes $\mathrm{R}$ phases from $\mathrm{T}$ phases by the $45^{\circ}$ difference in extinction angle. Any extinctions at angles other than $0^{\circ}$ (or $90^{\circ}$ ) and $45^{\circ}$ must be from the $\mathrm{M}$ or triclinic (Tri) phase domains. Note that all the phases except the Tri phase have 


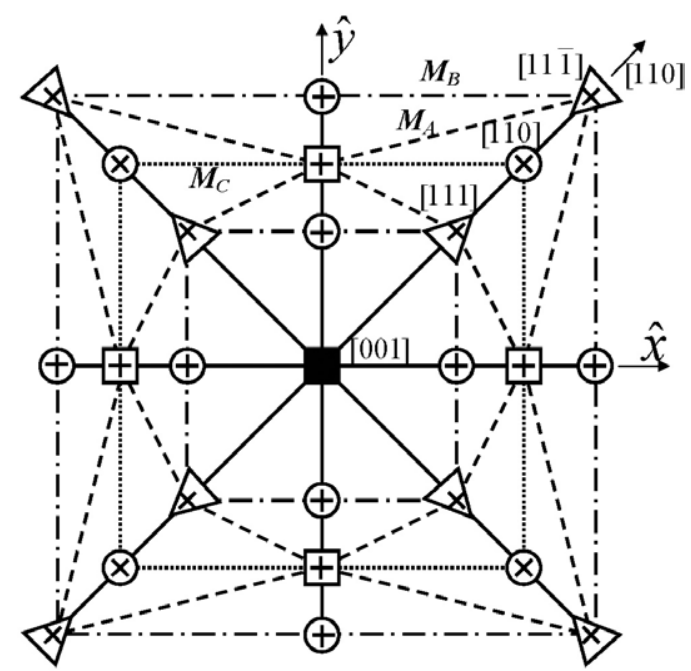

Figure 2. Relation between the optical extinction orientations corresponding to the ferroelectric polarization directions for various phases and domains projected on the (001) plane. Dashed, dashdotted, and dotted lines represent $M_{A^{-}}, M_{B}{ }^{-}$, and $M_{C^{-}}$type monoclinic phase domains, respectively. The definition of $M_{A}, M_{B}$, and $M_{C}$ phases can be found in [11] and [21].

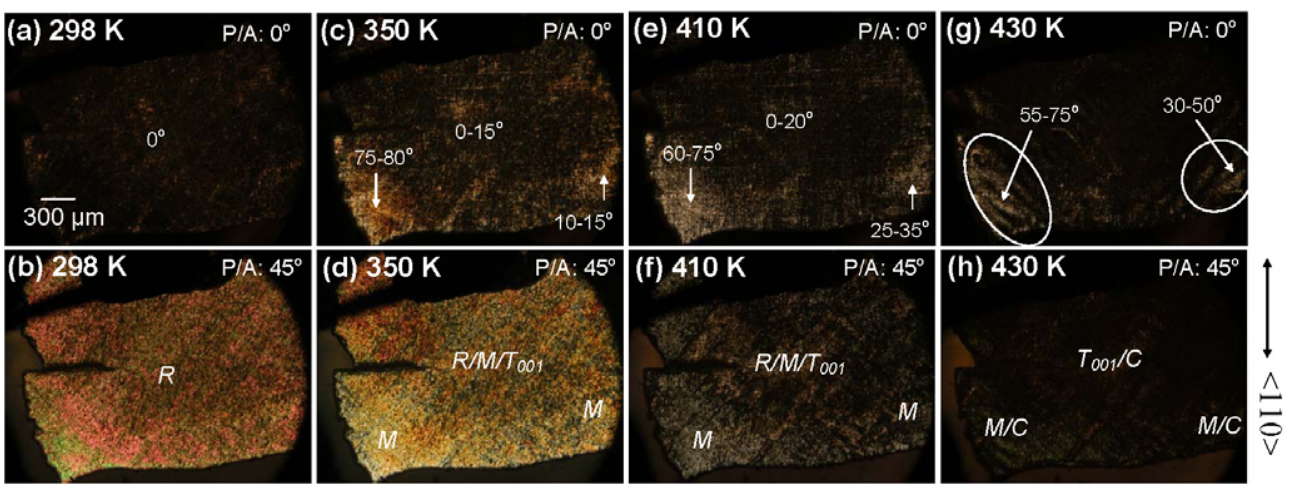

Figure 3. Temperature-dependent domain structures without any $E$-field poling. Domain micrographs taken at various temperatures with P/A: $0^{\circ}$ for the top row, and P/A: $45^{\circ}$ for the bottom row.

been reported in various PMNT crystals. Our large observed variation in extinction angle with $E$ field or temperature indicates $\mathrm{M}$ domains whose polarization $\boldsymbol{P}$ can vary with $E$ field through a large angle, whereas the polarization directions are nearly fixed for $\mathrm{R}, \mathrm{T}$, or $\mathrm{O}$ domains as the $E$ field or temperature varies.

\subsection{Thermal-induced phase transition}

What is the temperature-dependent phase transition in the unpoled PMNT33\% crystal? In figure 3, domain micrographs taken at various temperatures with P/A angle $=0^{\circ}$ are shown in the top row and P/A angle $=45^{\circ}$ are shown in the bottom row. As shown in figure 3(a), the domain matrix at RT $(298 \mathrm{~K})$ mostly exhibits extinction at $0^{\circ}$ with respect to the $\langle 110\rangle$ direction, indicating an $\mathrm{R}$ phase. If $\mathrm{O}$ or $\mathrm{T}$ phase domains exist, there will also be extinction 

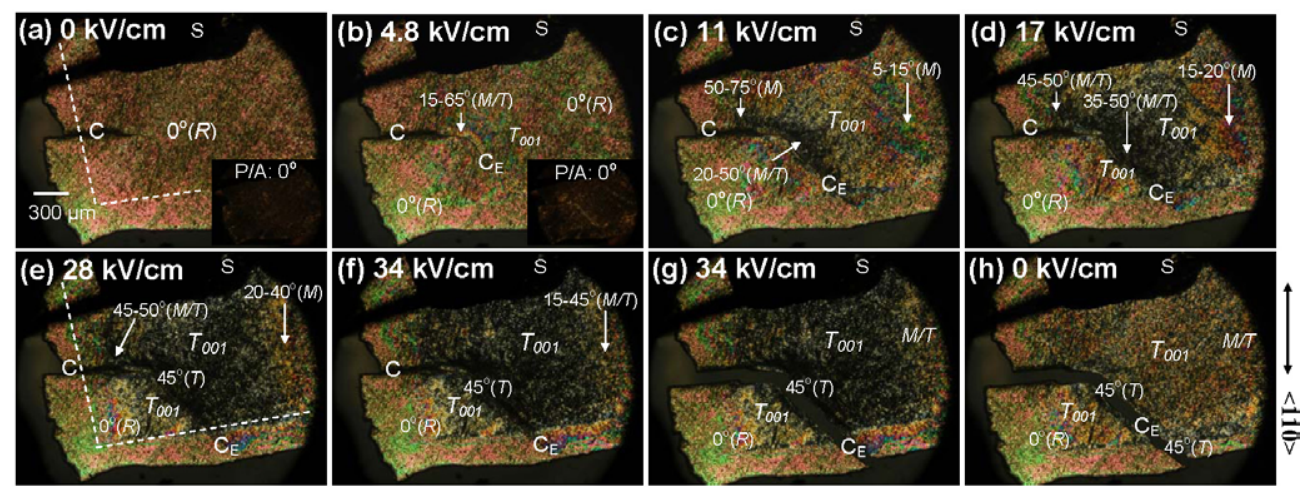

Figure 4. Domain micrographs taken with a P/A of $45^{\circ}$ (except the insets) at RT for a value of $E$ of (a) $0 \mathrm{kV} \mathrm{cm}^{-1}$, (b) $4.8 \mathrm{kV} \mathrm{cm}^{-1}$, (c) $11 \mathrm{kV} \mathrm{cm}^{-1}$, (d) $17 \mathrm{kV} \mathrm{cm}^{-1}$, (e) $28 \mathrm{kV} \mathrm{cm}^{-1}$, (f) $34 \mathrm{kV} \mathrm{cm}^{-1}$, (g) $34 \mathrm{kV} \mathrm{cm}^{-1}$, and (h) $0 \mathrm{kV} \mathrm{cm}^{-1}$ (right after the $E$ field is removed). The area in black indicated by ' $\mathrm{S}$ ' is the silver paste and the wire. The dashed lines show the ITO thin-film boundaries. ' $\mathrm{T}_{001}$ ' indicates the [001] tetragonal phase domain.

at P/A: $45^{\circ}$. However, no extinction was observed at P/A: $45^{\circ}$, as shown in figure $3(\mathrm{~b})$. As temperature increases to $343 \mathrm{~K}$, some of the domain matrix exhibits a continuous change in birefringence, shown as the brighter area in figure 3(c), which corresponds to an $\mathrm{M}$ phase. It indicates that the crystal has the coexistence of a dominant $\mathrm{R}$ phase and some minor $\mathrm{M}$ phase domains, i.e. R(M). Near $350 \mathrm{~K}$, the domain matrix shows an apparent change, which is consistent with the dielectric anomalies in figure 1. Some stripe-like domains display total extinction at all $\mathrm{P} / \mathrm{A}$ angles, which indicates a $\mathrm{T}_{001}$ phase, as shown in figure $3(\mathrm{~d})$. The $\mathrm{M}$ phase domain of the crystal at the corners exhibits extinctions in the ranges $10^{\circ}-15^{\circ}$ and $75^{\circ}-80^{\circ}$. As the temperature increases further, the domain area and the range of extinction angle for the $M$ phase expand, and the $\mathrm{T}_{001}$ phase domains also expand, as seen in figures 3(e) and (f). Near $430 \mathrm{~K}$, the $\mathrm{C}$ phase begins to appear as stripes with a total extinction at every P/A angle besides the $\mathrm{T}_{001}$ phase domains. In addition, a small portion of the domain matrix exhibits extinction angles of $55^{\circ}-75^{\circ}$ and $30^{\circ}-50^{\circ}$, as circled in figure $3(\mathrm{~g})$, indicating the $\mathrm{M}$ phase. Near $433 \mathrm{~K}$, the whole crystal becomes cubic phase. In brief, an $\mathrm{R} \rightarrow \mathrm{R}(\mathrm{M}) \rightarrow \mathrm{R} / \mathrm{M} / \mathrm{T}_{001} \rightarrow \mathrm{C}$ phase transition sequence takes place near 343, 350 and $430 \mathrm{~K}$ upon heating, respectively. $\mathrm{R}(\mathrm{M})$ indicates the coexistence of a dominant $R$ phase and some minor $M$ phase domains. $R / M / T_{001}$ indicates the coexistence of $\mathrm{R}, \mathrm{M}$, and $\mathrm{T}_{001}$ phase domains.

\subsection{E-field-induced phase transition and microcracking}

The $E$-field-dependent domain structures at RT, as shown in figure 4 , are taken at a P/A of $45^{\circ}$ while a dc $E$ field is applied along [001]. At $E=0 \mathrm{kV} \mathrm{cm}^{-1}$ (figure 4(a)), the domain matrix exhibits an $\mathrm{R}$ phase with extinction at a P/A of $0^{\circ}$, as shown in the inset. As the $E$ field increases, the domain matrix does not change apparently until $4.1-4.8 \mathrm{kV} \mathrm{cm}^{-1}$, as illustrated in figure 4(b). The domain matrix mostly still retains the $\mathrm{R}$ phase (inset of figure 4(b)), but $\mathrm{T}_{001}$ and $\mathrm{M} / \mathrm{T}$ phases were induced near the cracking area and exhibit extinctions at every $\mathrm{P} / \mathrm{A}$ angle and in the range $15^{\circ}-65^{\circ}$, respectively. M/T represents the coexistence of $\mathrm{M}$ and $\mathrm{T}$ phases with more $\mathrm{M}$ phase than $\mathrm{T}$ phase domains. Note that the coercive field $E_{\mathrm{C}}$ for this (001)-cut crystal is about $3 \mathrm{kV} \mathrm{cm}^{-1}$, as given in figure 5 . As the field increases to $11 \mathrm{kV} \mathrm{cm}-1$ (figure $4(\mathrm{c})$ ), the $\mathrm{T}_{001}$ phase domain significantly expands as crossed stripes along [100] and [010] from the cracking area. Some of the $\mathrm{R}$ phase domains have also transformed to $\mathrm{M}$ and 


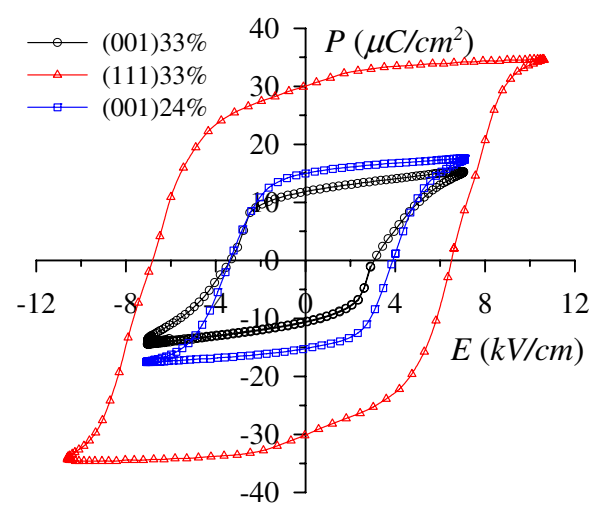

Figure 5. Hysteresis loops of polarization versus $E$ field obtained at RT from (001)-cut PMNT33\%, (111)-cut PMNT33\%, and (001)-cut PMNT24\% crystals. The results of (001)-cut PMNT24\% and (111)-cut PMNT33\% are inserted from [15] and [16], respectively, for comparison.

$\mathrm{T}$ phases with various nonzero-degree extinction angles, such as $5^{\circ}-15^{\circ}, 20^{\circ}-50^{\circ}$, and $50^{\circ}-$ $75^{\circ}$. But a small portion of the domain matrix still remains in the $\mathrm{R}$ phase, as indicated in figure 4(c). With increasing $E$ field, this $E$-field-induced transition is continuously evidenced by the expansion of the $\mathrm{T}_{001}$ phase domain, shown as a black area of the domain matrix in figures 4(d)-(f). At $E=34 \mathrm{kV} \mathrm{cm}^{-1}$, most of the domain matrix becomes a macroscopic $\mathrm{T}_{001}$ phase domain, except for some small $\mathrm{R}, \mathrm{T}$, and $\mathrm{M}$ phase domains with extinction angles of $0^{\circ}$, $45^{\circ}$, and $\sim 15^{\circ}-45^{\circ}$, as illustrated in figure $4(\mathrm{f})$. It is interesting that $\mathrm{T}$ phase domains usually appear near the cracks. In addition, a few domains exhibiting no extinction at any P/A angle are embedded in the $\mathrm{T}_{001}$ domain matrix, perhaps due to crystal defects and local strain caused by underlying $M$ phase distortions with several different orientations.

As indicated by ' $C_{E}$ ' in figure $4(\mathrm{~b})$, the microcracking caused by the dc $E$-field poling process starts to develop at $E \sim 4.8 \mathrm{kV} \mathrm{cm}^{-1}$ along [100] (or [010]) from the pre-existing crack ' $\mathrm{C}$ ' due to the polishing process. In a (001)-cut PMNT40\% crystal with an $E$ field applied along [001], we also found that microcracks are inclined to develop along [100] (or [010]) [17]. However, no microcracking was found in a (001)-cut PMNT24\% single crystal with $E=44 \mathrm{kV} \mathrm{cm}^{-1}$ applied along [001] at RT [15]. As shown in figures 4(c)-(f), the crack is enlarged by the increasing field strength. At $E=34 \mathrm{kV} \mathrm{cm}^{-1}$ (figure $4(\mathrm{~g})$ ), the crack reaches the sample edge and the crystal breaks into two pieces. The crystal still maintains the same R, $\mathrm{T}, \mathrm{T}_{001}$, and $\mathrm{M}$ phase domains as before it breaks (figure 4(f)). After the field is removed (figure 4(h)), the polarizing microscope picture becomes brighter than for $E=34 \mathrm{kV} \mathrm{cm}-1$ (figure $4(\mathrm{~g})$ ). The crystal does not return to an $R$ phase, as seen originally at zero field (figure 4(a)). Instead, the speckled appearance of the picture implies that $\mathrm{R}, \mathrm{T}_{001}, \mathrm{~T}$, and $\mathrm{M}$ phase microdomains coexist with numerous microdomains that have no extinction at any P/A angle, which perhaps is caused by the stress exerted by local polar microdomains of $\mathrm{M}$ phases with various orientations.

Figure 5 shows the polarization hysteresis behaviours versus $E$ field at RT for (001)-cut PMNT33\% (this work), (111)-cut PMNT33\%, and (001)-cut PMNT24\% crystals [15, 16]. In (001)-cut PMNT33\% crystal, the coercive field $E_{\mathrm{C}} \cong 3 \mathrm{kV} \mathrm{cm}^{-1}$, remanent polarization $P_{\mathrm{r}} \cong 12 \mu \mathrm{C} \mathrm{cm}^{-2}$, and spontaneous polarization $P_{\mathrm{S}} \cong 15 \mu \mathrm{C} \mathrm{cm}^{-2}$. The polarization hysteresis behaviour of the (001)-cut rhombohedral PMNT24\% crystal is similar to the (001)cut PMNT33\%. The (111)-cut PMNT33\% has a higher coercive field, $E_{\mathrm{C}} \cong 6.5 \mathrm{kV} \mathrm{cm}^{-1}$, and a much larger remanent polarization $P_{\mathrm{r}} \cong 30 \mu \mathrm{C} \mathrm{cm}^{-2}$, and spontaneous polarization 
$P_{\mathrm{S}} \cong 33 \mu \mathrm{C} \mathrm{cm}^{-2}$. The ratio between $P_{\mathrm{S}} \cong 15 \mu \mathrm{C} \mathrm{cm}^{-2}$ measured along [001] and $P_{\mathrm{S}} \cong$ $33 \mu \mathrm{C} \mathrm{cm}^{-2}$ measured along [111] at RT is fairly close to $1 / \sqrt{3}$. The deviation from $1 / \sqrt{3}$ may come from the different electrodes of silver paste and gold thin films for the (111)-cut and (001)-cut samples, respectively. $1 / \sqrt{3}$ is the projection fraction of the [111] rhombohedral polarization on the [001] axis. Note that the applied $E$ field in the hysteresis measurement of the (001)-cut PMNT33\% crystal is along [001]. This confirms that the PMNT33\% crystal is rhombohedral phase at RT. A (001)-cut tetragonal PMNT $x \%$ crystal has a much higher coercive field, $E_{\mathrm{C}}=8.25 \mathrm{kV} \mathrm{cm}^{-1}$, and a remanent polarization $P_{\mathrm{r}}=34.2 \mu \mathrm{C} \mathrm{cm}^{-2}$, where $x \%=39.4 \%$ is calculated with the formula we used for determining the Ti concentration stated in section 2 [20]. Therefore, the polarization hysteresis behaviour of PMNT crystals depends on the crystallographic orientation and symmetry (phase).

This (001)-cut PMNT33\% crystal cannot completely reach a $\mathrm{T}_{001}$ monodomain at $E=34 \mathrm{kV} \mathrm{cm}^{-1}$ applied along [001] at RT. In recent studies, a (111)-cut PMNT33\% crystal gradually reaches an $R_{111}$ monodomain at $E=12 \mathrm{kV} \mathrm{cm}^{-1}$ applied along [111] at RT [16]. A (001)-cut PMNT40\% single crystal becomes entirely $\mathrm{T}_{001}$ monodomain under $E=33 \mathrm{kV} \mathrm{cm}^{-1}$ applied along [001] [17]. However, a (001)-cut PMNT24\% single crystal cannot reach a $\mathrm{T}_{001}$ monodomain under $E=44 \mathrm{kV} \mathrm{cm}^{-1}$ applied along [001] at RT [15]. Therefore, a monodomain with orientation along the poling field was not always obtained under the maximum $E$-field strength in every crystal at RT as expected, such as the (001)cut PMNT24\% with $E$ field applied along [001] [15]. The $E$-field-induced monodomain can be achieved in the PMNT crystals if the dc poling field is along the polar axes of the phase favoured by the temperature, such as the (111)-cut PMNT33\% and (001)-cut PMNT40\% crystals $[16,17]$. A similar result was reported by Zhang et al that the $E$-field strength for the induced $\mathrm{M} \rightarrow \mathrm{T}$ phase transformation decays exponentially with increasing temperature in PMNT33\% crystals [13]. T polar directions are favoured at the higher temperature in PMNT33\% crystals, so that less $E$-field poling strength is needed to induce the T phase with the field applied along [001].

What is the physical origin of the $\mathrm{T}_{001}$ phase domains induced at RT by a dc $E$ field applied along [001] in PMNT crystals? In the (001)-cut PMNT24\% crystal, an R $\rightarrow \mathrm{T}_{001}$ phase transition was induced near $E=4 \mathrm{kV} \mathrm{cm}^{-1}$ through $M_{A}$ phase domains as the $E$ field increases along [001] at RT, i.e. $\mathrm{R} \rightarrow \mathrm{M}_{A} \rightarrow \mathrm{T}_{001}$ [15]. In the (001)-cut PMNT40\% crystal, the $\mathrm{T}_{001}$ phase domains were induced near $E=11 \mathrm{kV} \mathrm{cm}^{-1}$ at RT by the process of polarization rotation of $\mathrm{T} \rightarrow \mathrm{M} \rightarrow \mathrm{T}_{001}$, and this $\mathrm{T}_{001}$ phase expands through the whole crystal as the $E$ field increases further [17]. Similarly, in this (001)-cut PMNT33\% crystal the $\mathrm{T}_{001}$ phase domains were induced near $E=4.1 \mathrm{kV} \mathrm{cm}^{-1}$ at RT and expand significantly at $E=11 \mathrm{kV} \mathrm{cm}^{-1}$ by various phase transition sequences $R \rightarrow \mathrm{T}_{001}, \mathrm{R} \rightarrow \mathrm{M} \rightarrow \mathrm{T}_{001}$, $\mathrm{R} \rightarrow \mathrm{T} \rightarrow \mathrm{T}_{001}$, and $\mathrm{R} \rightarrow \mathrm{M} \rightarrow \mathrm{T} \rightarrow \mathrm{T}_{001}$.

\section{Conclusions}

In this unpoled (001)-cut PMNT33\% sample, a first-order-type $\mathrm{R} \rightarrow \mathrm{R} / \mathrm{M} / \mathrm{T}_{001} \rightarrow \mathrm{C}$ phase transition sequence takes place near 350 and 430 , respectively, upon heating. Under the $E$-field application along [001], the [001] tetragonal $\left(\mathrm{T}_{001}\right)$ phase domains were induced by various phase transition sequences, i.e. $\mathrm{R} \rightarrow \mathrm{T}_{001}, \mathrm{R} \rightarrow \mathrm{M} \rightarrow \mathrm{T}_{001}, \mathrm{R} \rightarrow \mathrm{T} \rightarrow \mathrm{T}_{001}$, and $\mathrm{R} \rightarrow \mathrm{M} \rightarrow \mathrm{T} \rightarrow \mathrm{T}_{001}$. The $\mathrm{T}_{001}$ phase domains expand through the whole crystal via the $M$ phase with increasing $E$ field. The intermediate $M$ phases play an essential role in bridging higher symmetries while the $E$-field-induced transitions are taking place. This confirms our previous findings in (001)-cut PMNT24\%, (111)-cut PMNT33\%, and (001)-cut PMNT40\% crystals [15-17]. By comparison with our previous results in PMNT $x \%$ ( $x=24 \%, 33 \%$, 
and 40\%) [15-17], we found that whether or not a single domain can be induced by a dc $E$ field strongly depends on the crystal temperature, i.e. it depends on whether the polar axis of the induced single domain is favoured by the temperature. In addition, we also found that $E$ field-induced microcracks are inclined to develop along [100] (or [010]), which is the growth direction of the $\mathrm{T}_{001}$ phase domain that is being induced by a dc $E$ field applied along [001].

\section{Acknowledgments}

The authors would like to express their sincere thanks to Dr H Luo for the crystal. This work was supported by US Department of Defense (DoD) Experimental Program to Stimulate Competitive Research (EPSCoR) Grant No. N00014-02-1-0657 and National Science Council (NSC) Grant No. 94-2112-M-030-004.

\section{References}

[1] Kuwata J, Uchino K and Nomura S 1981 Ferroelectrics 37579

[2] Choi S W, Shrout T R, Jang S J and Bhalla A S 1989 Ferroelectrics 10029

[3] Shrout T R, Chang Z P, Kim N and Markgraf S 1990 Ferroelectr. Lett. 1263

[4] Noheda B 2002 Curr. Opin. Solid State Mater. Sci. 6 27-34

[5] Singh A K and Pandey D 2003 Phys. Rev. B 67064102

[6] Shuvaeva V A, Glazer A M and Zekria D 2005 J. Phys.: Condens. Matter 175709

[7] Park S-E and Shrout T R 1997 J. Appl. Phys. 821804

[8] Park S-E and Hackenberger 2002 Curr. Opin. Solid State Mater. Sci. 611

[9] Han P, Yan W, Tian J, Huang H and Pan H 2005 Appl. Phys. Lett. 86052902

[10] Fu H and Cohen R E 2000 Nature 403281

[11] Davis M, Damjanovic D and Setter N 2006 Phys. Rev. B 73014115

[12] Tu C-S, Chien R R, Wang F-T, Schmidt V H and Han P 2004 Phys. Rev. B 70220103

[13] Feng Z, Luo H, Guo Y, He T and Xu H 2003 Solid State Commun. 126347

[14] Zhang S, Luo J, Xia R, Rehrig P W, Randall C A and Shrout T R 2006 Solid State Commun. 13716

[15] Chien R R, Schmidt V H, Tu C-S, Hung L-W and Luo H 2004 Phys. Rev. B 69172101

[16] Tu C-S, Schmidt V H, Chien R and Shih I-C 2003 Appl. Phys. Lett. 831833

[17] Chien R R, Schmidt V H, Hung L-W and Tu C-S 2005 J. Appl. Phys. 97114112

[18] Tu C-S, Schmidt V H, Shih I-C and Chien R 2003 Phys. Rev. B 67020102

[19] Schmidt V H, Chien R, Shih I-C and Tu C-S 2003 AIP Conf. Proc. 677160

[20] Hu C, Fang B, Luo H, Sun Y and Guo J 2003 Ceram. Int. 29145

[21] Vanderbilt D and Cohen M H 2001 Phys. Rev. B 63094108 